\title{
The Role of Computational Science and Emerging Technologies in the Natural Sciences Education at University Level
}

\author{
Nia Alexandrov ${ }^{\mathrm{a}}$,Vassil Alexandrov $^{\mathrm{b}}$ and Raul Ramirez ${ }^{\mathrm{c}}$ \\ ${ }^{a}$ Barcelona Supercomputing Centre, Barcelona,Spain and School of Systems Engineering, University of Reading, UK \\ ${ }^{b}$ ICREA and Barcelona Supercomputing Centre, Barcelona, Spain \\ ${ }^{c}$ ITESM, Monterrey Tech, Monterrey, Mexico
}

\begin{abstract}
This paper is focused on the role of Computational Science and emerging technologies in the natural sciences education at university level. We outline our Integrated Metacognitive Process Model (IMPM) and our Collaborative Learning approach based on Collaborative Creative Cross-Pollination activity model at postgraduate level. We present our multidisciplinary approach based on the following three components: the existence of multidisciplinary research environment (non-silos departmental culture), computational science research methods as core part of the curricula and collaborative teaching activities facilitated by novel collaborative tools using Collaborative Creative Cross-Pollination. Some results showing the advantages of such an environment and approach are presented. The initial results have shown overall average improvement of the average marks with around $5 \%$ plus clear satisfaction of the students as evident from their responses to the course evaluation.
\end{abstract}

Keywords: Computational Science education, postgraduate programs, multidisciplinary education, collaborative learning.

\section{Introduction}

This paper focuses on the role of Computational Science and emerging technologies in the natural sciences education at university level. The gap between the high-level science and technology directives, and a detailed Computational Science curriculum under the unifying concept of Computational Science for Exascale is identified. The following is a gap analysis of international Computational Science education and graduate curriculum: In Europe, there are relatively few MSc. programs in Computational Science, and related areas such as

* Corresponding author. Nia Alexandrov, Tel.: +34 934137513.

Authors e-mail addresses: nia.alexandrov@bsc.es; vassil.alexandrov@bsc.es; rramirez@itesm.mx.

Nia Alexandrov is HPC Professional Training and Postgraduate Studies Coordinator at BSC, Spain, she held previously Postgraduate Studies and Professional Training Coordinator position at ACET (Advanced Computing and Emerging Technologies) Centre, School of Systems Engineering, University of Reading, UK and was coordinator of 3 MSc programs: MSc in Network Centred Computing, Erasmus-Mundus MSc in Network and E-Business Centred Computing and MSc in Computational Science by research, she is currently completing her PhD studies at the University of Reading, UK 
Computational Science and Engineering, Scientific Computing, High Performance Computing, and Supercomputing. Programs include University of Amsterdam Netherlands (MSc in Computational Science), The University of Manchester (UK), École polytechnique fédérale de Lausanne, ETH Zurich (Switzerland), KTH, and Uppsala University (Sweden), RWTH Aachen, TU Darmshtadt, TU Munchen, University of Erlangen (Germany), UPC/BSC (Spain), and Helsinki University (Finland).

In the rest of the world, the concentration of such programs is mainly in USA and Canada. Leading programs are found at: George Mason University, Georgia Tech, Mississippi State University, New York University, Ohio University, Pennsylvania State University, Princeton University, Stanford University, State University of New York, San Diego State University, University of California, University of Houston, University of Iowa, University of Illinois, University of Utah (USA), Memorial University, University of Toronto, Queen's University, McMaster University, and University of Waterloo (Canada).

Outside Europe and North America, the key universities offering Computational Science programs are National Singapore University (Singapore) and Seoul University (Korea).

Europe seems to lag behind North America both in the number and in the variety of Computational Science programs offered. Analyzing the curriculums of current European MSc. programs in Computational Science and related areas, we observe that they tend to fall into two categories. Either they focus on a particular application area (such as Engineering, Biology, etc.), or they focus on computer science techniques for high performance or scientific computing. In general they often fail to balance the core technical knowledge in high performance computing with appropriate domain or application knowledge. In addition, there is also a technical skills gap between their curriculum and key applications in industry.

Gartner, a consulting and research company in the field of ICT (Information and Communications Technology), has identified seven technologies that will disrupt ICT within the next 25 years (http://www.bulletinselectroniques.com/actualites/54036.htm). These challenges are portrayed by Gartner as "basic problems to which solutions will be made in the ICT field and that will have enormously beneficial effects on the economy, science and society in all aspects of daily life." One of these major challenges is parallel computation: "Advances in computing speed have started with multicore processors that use multiple processors to solve problems faster by dividing tasks into smaller individual processes. The challenge is to design applications that take advantage of multicore processors in determining which tasks can be performed simultaneously by different processors."

Recently, Exascale Computing, an attempt to harness thousand fold projected increases in computational power, has emerged as a Grand Challenge research area. This highlighted the need "to advance fundamental research in Computational Science and Engineering and in fundamental, applied and interdisciplinary mathematics and statistics" [3-5]. It has been observed that "beyond accelerating disciplinary progress, investments in these fields are needed to drive discovery in every science and engineering discipline and to power the use of next-generation cyberinfrastructure and networking". Meeting the Exascale Computing Challenge requires a sustained effort in key areas of Computational Science ranging from the development of novel multi and many-core architectures, to new programming models, scalable algorithms, and new modeling techniques and paradigms . Computational Science has been identified as being "crucial for the development of science" [3], and graduate level education must play an important role in its development.

On the other hand an IDC (International Data Corporation) talent study in 2010 [1] has identified the major inflection points: Parallelism, and how to use it, Petascale/exascale computing, HPC system heterogeneity, HPC system architectural balance, HPC system reliability, HPC system and data center power and cooling. Skills most difficult to find to tackle the inflection points are: Scientists with HPC capabilities ("Combined scientific background and HPC programming skills", "Computational scientists" ), Parallel Programmers ("Experience in parallel software development", "Engineers and scientists that can program in HPC/parallel Fortran" , "Parallel code porting/optimization"), Algorithm Developers,("For computational science people who can help researchers develop and implement new algorithms"), System Administrators With High-End Computing Experience 
"Scientific computing system management experience", "System administrators with HPC expertise")[1].

In the USA there are multiple efforts at several levels to tackle the gap: XSEDE is the Extreme Science and Engineering Discovery Environment project [6] funded by NSF with the most advanced and robust collection of integrated digital resources and services in the world with strong education and outreach programs in Computational Science. Also at College and Undergraduate level a focused and consistent effort is made by SHODOR [7] in the past decade to promote Computational Science and introduce novel pedagogical approaches.

Within that context, and despite of several clusters of excellence, overall Europe lags behind and really concerted efforts are needed to bridge the gap. In this paper we would like to share our approach, experience and expertise into tackling the gap at graduate (postgraduate) level.

\section{Multidisciplinary Education}

In the past decate with the advances in various areas Computational Science became a key for the advancement of Science. As highly multidisciplinary by nature it requeres different approaches in order to deliver effective education and produce the right learning outcomes.

Currently we are also observing a paradigm shift towards muldidisciplinary education. This stems from the major drivers such as Energy, Climate Change, Urbanization etc [2], to cite a few, and the fact that these challenges really require multidisciplinary approach and knowledge to tackle these challenges.

Analysing the the existing approaches and programs we can outline five major steps:

Step one: use of IT as individual user

Step two: Training for Collaborative Work

Step three: Web 2.0 tools for support of collaborative work

Step four: Integration in the subject matter of

- High Performance Computing and HPC Domain Specific Applications

- Visualisation \& Simulation

Step five: Complex Systems Approach to Problem Solving

- Mathematical Modelling \& Problem Solving

- Cross Disciplinary Collaborative Learning Activities

Considering Steps one to three, it is evident that here gradualy we move from facilitation of IT for individual user on Step1 to facilitation of collaborative learning at Step3 using novel technologies. These steps can be considered as facilitation steps.

Step four goes further and enables to integrate material in the subject matter, for example, high performance computing techniques, novel mathematical modelling approaches, visualization and advanced simulation techniques.

And the final and most important step is building the true multidisciplinary curricula, where we have a Complex Systems Approach to Problem Solving with focus on Mathematical Modelling \& Problem Solving (with domain specific examples) as a multidisciplinary team through Cross Disciplinary Collaborative Learning Activities.

In our case the IT training of the individual user for the software to be used is done as a training course at the start of the MSc programs. Training for collaborative work is embedded as part of the Transferable and Research Skills modules of the MSc programs. Collaborative Computing and the introduction to key Web2.0 tools is made in Network Computing module. Cross-disciplinary Collaborative activities are part of the Network Computing module and also involve a team project. And finally High Performance Computing, Visualisation and Simulation are given as separate modules as well as advanced components of those being embedded in some of more application focused modules. 


\section{Pedagogical Approach and Methodology}

\subsection{Integrated Meta-cognitive process model}

Our Integrated Meta-cognitive process model [9] is given on Figure 1. It has the following five components:

- Knowledge domain, which not only describes the knowledge that we desire students to acquire, but also certain different skills, which can be cognitive, affective and motor. This component represents the main goals and objectives of the educational process. Cognitive skills enable students to correctly mentally organize knowledge so that it can be selected and used effectively, to differentiate or induce knowledge such that it can be applied in different contexts, or to integrate knowledge or deduce knowledge so it can be restructured to fit new situations. Creativity, the ability to create new knowledge is also an important part of the knowledge domain and should always be considered as part of the educational program. It is important to point out that knowledge by itself can also be classified, according to some authors [8] as conceptual, procedural and metacognitive. This classification is important, as students must construct knowledge in increasing phases of complexity. When information technology is involved, other skills will be acquired by students such as ability to work in different scenarios with different types of information, the possibility to use different information channels to interpret phenomena, ability to communicate synchronously and asynchronously, ability to take responsibility in decision making, ability to refine information selection criteria, etc.

- Educational subjects. It indicates the nature and origins of students and teachers and the relationship between them. It is important to recognize the age group, academic background and other culture related information about students. Effective learning is achieved when the process is focused on student's interests, culture and environment, and when new knowledge and skills are constructed upon a well identified academic base. The location and available resources to learners in such location is also very important as it will influence which learning activities students can carry out. The relationship between teachers and students is also important. Learning can be a self-directed experience where the teachers role is more that of a tutor than that of an authority. In such a relation, students have a large degree of control on their learning and power is more balanced with that of the teacher. On the other hand, learning can be a much directed experience, as in more traditional learning. In this case, teachers have most of the control of the learning process as well as most of the power. Self-directed learning experiences mediated by information technology (distance learning) require more planning and material preparation from teachers. Thus materials, tools and the learning process as a whole must be very easy to use and understand.

- Contexts and environments. They establish the variables that make-up the virtual learning space. The same knowledge domain may be taught to different people with different culture, in different locations with different resources, and at different times with different emphasis, by different teachers with different types of communication. Thus the analysis of contexts tries to identify those variables and how they affect learning in order to be able to reuse as much as possible the already developed activities and materials and to make the learning process more effective. Contexts are very important, as the most effective materials and activities are those that focus on the student's background, interests, culture and environment.

- Educational processes. In general, this term identifies the activities that must be carried out by students in order to acquire skills and knowledge. Those activities are usually designed following certain philosophies such as conductivism, cognitivism and constructivism. They take into account the knowledge domain, student's background and learning contents. Those activities may be such as searching, reading, viewing videos, hearing audio, dialog and discussion, drill and practice, problem solving, research, quiz solution, reflexion, write essays, etc. They are structured in such a way that students progress from simple to complex knowledge and skills, to knowledge creation. They are varied enough so students can use different 
channels to acquire knowledge depending on the inner workings of their brain and body. Also, different paths can be taken in order to achieve learning goals. Constant feedback and evaluation is carried out, and different students may achieve learning objectives at different speed.

- Digital content and technology. Also based on the knowledge domain, student's background and the learning context, digital contents and technology are closely tied to the educational processes for basic learning activities that are carried out using them. Digital contents and technology must endeavor to target as many communications channels as possible as well as students' different intelligence capacities such as linguistic, visual, kinesthetic, musical, logical-mathematic, interpersonal, etc. Digital contexts may vary according to the type of knowledge they support; conceptual, contextual and procedural. For example, we can use graphics and 3D animations to show the meaning of certain concepts and the relation between entities and components. We can use video and audio to show procedures and human interaction. We can use interactive simulations to help understand how different environments modify a system's behavior under certain contexts, etc. But digital content not only need to support knowledge acquisition, but also cognitive skills, attitudes and values. This can be done by showing different scenarios and different outcomes to carefully planned hypothetic situations and by promoting conversation, argument, debate and discussion. This is usually carried out through a special technology, a software program called the learning management system (LMS). The role of such system is to support file storage and delivery, information sharing, communication, planning, statistics recollection, discussion and dialog, assessment and evaluation for individuals, and all that plus collaboration for teams and groups.

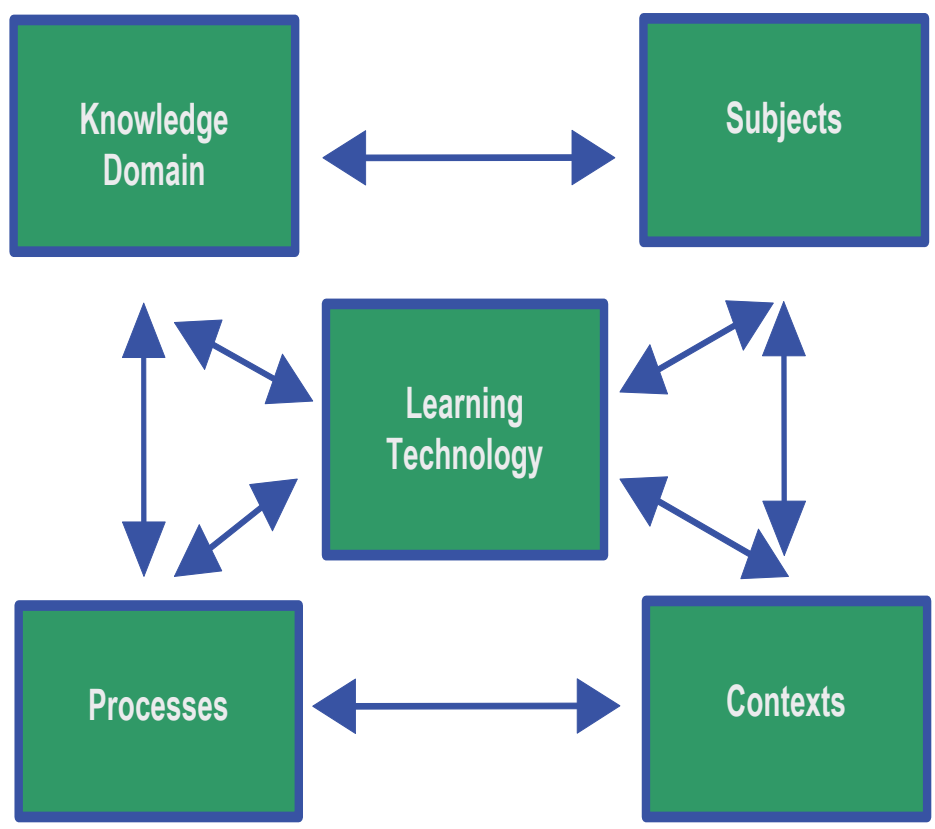

Fig. 1. Integrated Metacognitive Process Model (IMPM) 


\subsection{Meta-cognition and collaborative learning}

Our collaborative learning approach is based on the Integrated Metacognitive Process Model. Our successful collaborative activity models establish the relation between the different activity stages in the Integrated Learning Processes [9], but also establish the metacognitive blueprint. Based on the Integrated Metacognitive Processes Model (IMPM) we establish a range of goals, conditions and boundaries that will differentiate the activity model from others (See [9] for more information on the IMPM). Figure 2 presents the relationship between the Collaborative Learning based on Collaborative Creative Cross-Pollination (CCCP) activity model and the IMPM model.

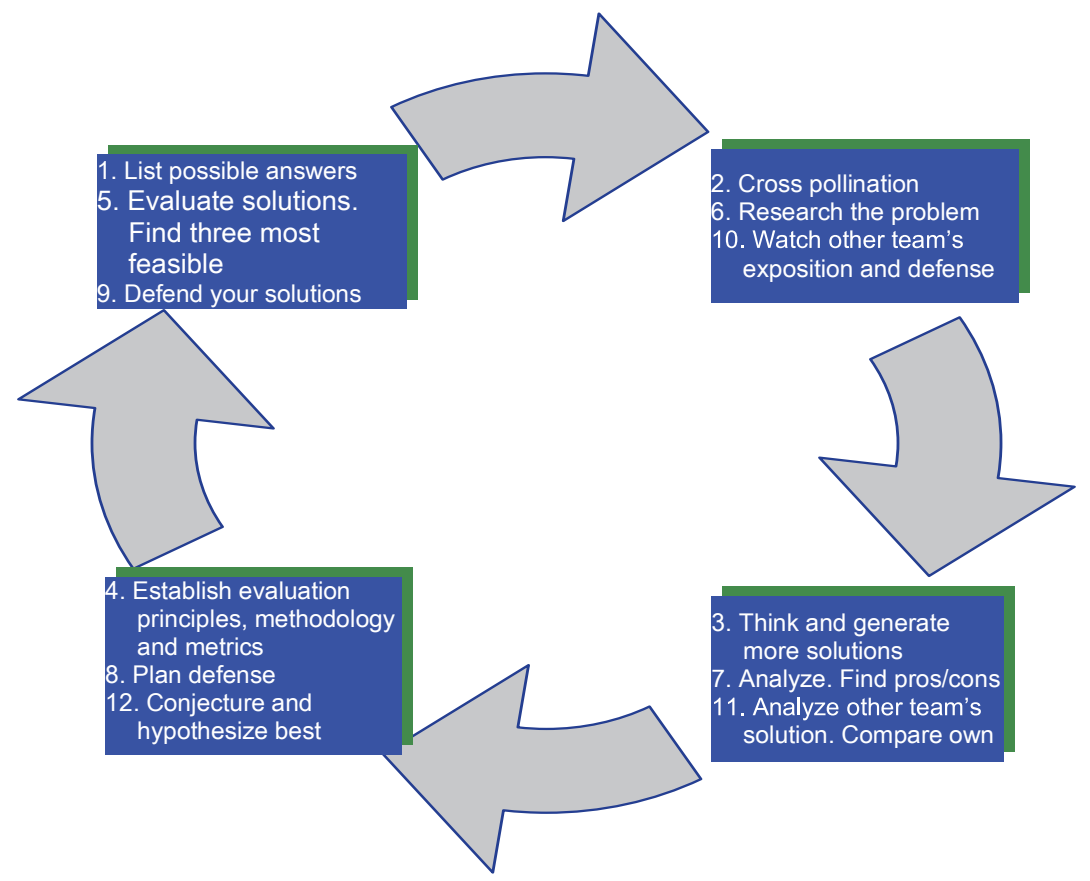

Fig. 2. Relation between the Collaborative Creative Cross-Pollination and the IMPM model

In our approach in a nutshell:

- Students are introduced to team work on Transferable Skills or Research Skills module.

- Students are introduced to collaborative computing and understand how to use collaborative tools on the Network Computing Module.

- In terms of Context:

- The Teacher's/Lecturer's and the Tutor's roles have changed from lecturer to facilitator \& mediator during collaborative learning activities (Salmon's model [10]) $+$

- Right cognitive complexity technical assignment is given

- Resulting in challenging tasks to be tackled allowing testing of student's ideas/ concepts. 
- Students perform advanced technical team assignment implemented through a team project in Network Computing module based on our Collaborative Creative Cross-Pollination activity model.

The team assignment is designed in the following way:

- Separate teams are formed taking into account the technical background and the research interests of the student, and also have students with different backgrounds.

- The assignment is given in a form of a topic with an initial scenario with given parameters, which students develop further as a team (hence developing and enhancing their research skills).

- Students are encouraged to use previous research assignments relating to the topic given in the literature review phase (from Transferable Skills and Research Skills modules).

- Crosspollination exercises are set in the beginning (where each team presents their approach, work progress and expected results), midterm (when the session is considerably longer and cross-team cooperation is organized in the following fashion: from each team students with the same team role are put together to discuss major issues and find solutions (for example, if the aim of the team project is to create a VO, each team will have a scenario from different application area and in the midterm crosspollination exercise the discussion groups are built, for example, around the areas of HPC, algorithms, mathematical methods and models to be used, software systems issues, security, organizational support by collaborative tools.) After the end of the discussion the results are integrated into the work of the individual teams.) and at the end of the project, after the production of the team report, when the final presentation of each team is discussed. The students are encouraged to comment on: the methods of decision making employed and the level of collaboration of their team, on the effectiveness and usefulness of midterm crosspollination exercise

The initial results have shown overall average improvement of the average marks with around 5\% plus clear satisfaction of the students as evident from their responses to the course evaluation. It was also observed a clear improvement of lower performing students by improving the quality of their individual components of work within the team project showing better grasp of complex concept and more confident application of problem-solving techniques.

\subsection{Multidisciplinary approach}

On Postgraduate level, for student projects and project related teaching in order to be successful in this new set of skills requirements, it depends on the following three components (see Figure 3): the existence of multidisciplinary research environment (non-silos departmental culture), computational science research methods as core part of the curricula and collaborative teaching activities facilitated by novel collaborative tools.

Our experience of individual projects on $\mathrm{PhD}$ level between life and natural sciences and Computational Science based on the above approach proved to be successful. 


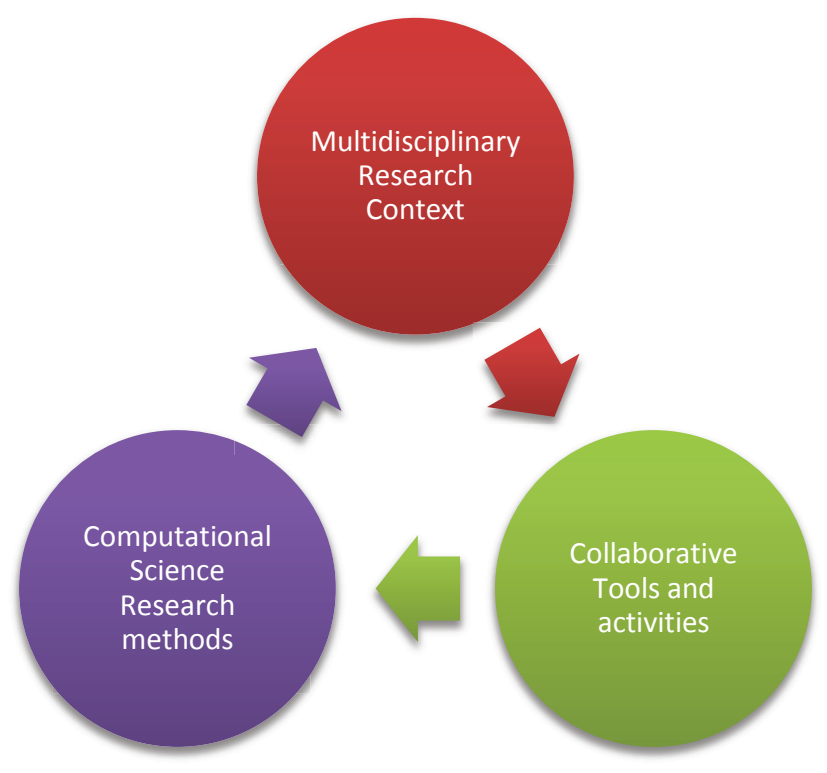

Fig. 3. Research Environment

In most of the cases this is not true because one major problem of recognition and truly multidisciplinary approach in Europe is the individual assessment by various Research Boards and Bodies as well as the research returns, all these are quite departmentalized and count much more on disciplinary achievements and metrics than on multidisciplinary ones. In addition, very often Computational Science and Supercomputing are treated as service provider to other sciences and not as what they really are - a significant research areas and sciences on their own and should be treated as an equal research partner in any multidisciplinary collaboration. We believe that our approach provides a truly multidisciplinary setting.

\section{Results}

We have implemented our approach at MSc level to the Erasmus-Mundus MSc in Network and E-Business Centred Computing, MSc in Network Centred Computing and MSc in Computational Science by Research. The first one is a joint degree awarded by three Universities, and the last one is a double degree awarded by two Universities. All these require very efficient use of IT in order to facilitate the degrees delivery.

The survey of the students have revealed the following results:

1. Positive experiences as indicated by students :

- $\quad 24 / 7$ availability and remote access to data and content, as access to e-books, articles, forums, wikis etc.

- Access to collaborative tools to exchange information, collaborate on projects, ability to collaborate at any time and at distance.

- Learned many new technologies which can be used in so many attractive areas.

2. Over $90 \%$ were satisfied and very satisfied using BlackBoard, and collaborative tools such as GoogleDocs, Chat room provided, Group e-mail, Wiki, Forum, etc. 
3. The teaching methods used based on the pedagogical approach outlined above were rated also high:

- Over $80 \%$ positive and very positive satisfaction of the Content and assessment of the programs.

- Over $85 \%$ positive and very positive satisfaction of the student involvement and interaction with the lecturers, tutors and professors as well as over $85 \%$ satisfaction of the teaching and Learning resources provided.

4. Important contributing factors being for example:

- Lab access $24 / 7$

- Good interaction with professors and good IT resources provided significant learning support

- Good learning environment

- Involvement of Teaching Assistants and interaction with the alumni was very useful

- International experience.

5 Over $80 \%$ outlined the importance of reliability of the technology, software to be up to date and reliability of storage media

6 Over $80 \%$ rated the Courses organization, teaching methods and student support as good and very good, very structured and balanced, block and intensive system of lecture/labs as being very beneficial, very well organized.

7 Social networking site mainly used by students firstly for socializing and secondly for study was Facebook,

8 Collaborative tools used primary for study and work and secondary for socializing were equally at first place YouTube and Wikis and in second place Blogs and GoogleDocs.

The overall result has shown an average of 5\% increase of achievements and marks of the students by applying our $\mathrm{CCCP}$ approach in comparison with the previous intakes.

One student statement summarizes it all:

The program "is pushing students to the limit properly understanding how to reach goals through proper research"

Our experience shows that in order to be successful in implementing Computational Science and Computational Science related programs at graduate and postgraduate level several important elements are needed to be present:

- Existence of non-silos departmental culture, supported by joint and cross-disciplinary examination boards.

- Ensuring multidisciplinary postgraduate curricula by enforcing a minimum of required credits outside of the student's narrow specialization.

- Focusing on Research Methods and Skills that could be achieved by credited and assessed taught modules.

- Exposure to collaborative work in multidisciplinary setting through implementation of team coursework with problems taken from application areas.

- Significant final research project (of no less than 30 ECTS) with structured requirement for novel research element and publishable result, not just development or re-development project.

- Exposure and access on postgraduate level to HPC (High Performance Computing) resources and acquiring the necessary basic skills to use them.

\section{Conclusion}

In this paper we present some results of applying Collaborative Learning based on Collaborative Creative CrossPollination (CCCP) activity model at postgraduate level. We also outline the importance of the following three components: the existence of multidisciplinary research environment (non-silos departmental culture), computational science research methods as core part of the curricula and collaborative teaching activities facilitated by novel collaborative tools using CCCP. 
In such an environment the initial results have shown overall average improvement of the average marks with around 5\% plus clear satisfaction of the students as evident from their responses to the course evaluation. It was also observed a clear improvement of lower performing students by improving the quality of their individual components of work within the team project showing better grasp of complex concept and more confident application of problem-solving techniques. Our experience at doctoral level has been also positive.

We are applying the above approach to new joint MSCs and Doctoral programs currently in a process of curricula design and program development as well as investigating the use of the same approach into professional training of researchers and systems specialist as part of the training program of PRACE advanced teaching centers [11].

\section{Acknowledgements}

The authors are grateful to their MSc in Network Centred Computing, Erasmus-Mundus MSc in Network and eBusiness Centred Computing and MSc in Computational Science by Research students in participating in the surveys.

\section{References}

1. Earl C. Joseph, Steve Conway, Jie Wun, IDC Tallent StudyA Study Of The Talent And Skill Set Issues Impacting HPC Data Centers Conducted On Behalf On The US Department Of Energy (2010).

2. Dr Chris Luebkeman and the Foresigght team at ARUP (Jennifer Greitschus eds.), Prestel Verlag and Arup, 2009, ISBN 978-3-79134224-5

3. A Strategy for American Innovation: securing our economic growth and prosperity, National Economic Council, Council of Economic Advisers, and Office of Science and Technology Policy February 2011.

http://www.whitehouse.gov/sites/default/files/uploads/InnovationStrategy.pdf

4. Modelling and Simulation at the Exascale for Energy and the Environment, US Office of Science.

5. The international exascale computing project,, http://hpc.sagepub.com/content/23/4/309.sh

6. XSEDE - Extreme Science and Engineering Discovery Environment project, https://www.xsede.org/

7. Shodor a national resource for Computational Science Education, www.shodor.org/

8. Bloom, B. S. "Taxonomy of educational objectives: The classification of educational goals: Handbook I, cognitive domain". New York, 1956. Longmans, Green.

9. N.S. Alexandrov, R.V. Ramirez-Velarde, The Integrated Learning Process, Metacognition and Collaborative Learning, Proc. of Interactive Computer-aided Learning, Villach, Austria, 2007.

10. Gilly Salmon, E-moderating:the key to teaching and learning online, Routledge Falmer, $2003-242$ p.

11. PRACE - Partnership for Advanced Computing in Europe, www.prace.eu 